\title{
Contributions des intermédiaires de transition à la diffusion d'une niche sociotechnique
}

\section{Contributions of Transition Intermediaries in Sociotechnical Niche}

Diffusion

\section{Carola Guyot Phung ${ }^{1}$}

${ }^{1}$ Laboratoire i3 - CRG, Ecole Polytechnique - Paris Saclay, France, carola.phung@laposte.net

Cette recherche a été rendue possible grâce au financement de l'Agence de l'Environnement et de la maîtrise de l'Energie (ADEME) et de la Fédération Professionnelle des Entreprises du Recyclage (FEDEREC).

RÉSUMÉ. La transition vers un modèle de société durable peut être impulsée grâce à des politiques publiques qui privilégient l'innovation comme voie de transformation. Cela passe par la création d'espaces dédiés à l'innovation et à l'expérimentation, les niches sociotechniques, qui constituent une solution pour faire germer un changement. Cet article propose de caractériser les intermédiaires de transition, et de mettre en évidence leur rôle dans le développement et la diffusion d'une niche sociotechnique. A travers l'étude de deux cas d'innovation du secteur du recyclage en France, l'une émergeant de la réglementation, l'autre volontaire, nous montrons que le développement et la diffusion d'une innovation peut s'appréhender à travers une chaîne d'intermédiaires de transition. Parmi eux, les intermédiaires de niche jouent un rôle crucial dans ce processus.

ABSTRACT. The transition to a sustainable social model can be fostered by policies that nurture innovation as a path to transformation. This involves spaces dedicated to innovation and experimentation, sociotechnical niches, which can be purposively created to generate change. This article highlights the characteristics of transition intermediaries and their contribution to niche development and diffusion. Two case studies from the French recycling industry are analyzed. Transition intermediaries play complementary roles within a chain, and niche intermediaries appear as a central element in niche development and diffusion.

MOTS-CLÉS. Transition environnementale, niche sociotechnique, intermédiaires de transition, innovation. KEYWORDS. Sustainability transition, sociotechnical niche, transition intermediaries, innovation.

\section{Introduction}

La transition vers un modèle durable de société peut être impulsée et pilotée grâce à des politiques publiques qui privilégient l'innovation comme voie de transformation. Nous situons notre travail dans le cadre de l'approche sociotechnique de la transition [GEE 02]. Elle envisage cette dernière comme une innovation systémique entre trois niveaux : le paysage, le régime sociotechnique et la niche sociotechnique. Cette dernière est un espace créé pour initier le changement et dédié à l'innovation. Des projets concrets peuvent s'y développer, protégés de la sélection du régime sociotechnique. Mais favoriser le changement implique une approche systémique de l'innovation [VAN 03], et les intermédiaires de transition [KIV 14] engagés dans ce processus peuvent y contribuer. 
Cette figure récente de la transition reste encore peu étudiée alors que son rôle peut être structurant dans le développement d'une niche [GEE 06a] [KIV 14]. Des travaux récents [KIV17] ouvrent la voie à une meilleure compréhension de ces acteurs, et nous proposons d'approfondir leur caractérisation et leur rôle dans le développement d'un projet d'innovation [MAR 18].

Après avoir présenté l'approche sociotechnique de la transition et les intermédiaires de transition, nous abordons la méthodologie. Dans une quatrième partie, deux cas distincts d'innovation, issus du secteur du recyclage, sont présentés et discutés. Nos contributions viennent en cinquième partie.

\section{Cadre théorique}

\subsection{L'approche sociotechnique de la transition}

L'approche sociotechnique de la transition (GEE 02, 11] présente les phénomènes de transitions comme un modèle de diffusion de l'innovation à travers un système social. Le changement découle de l'interaction entre trois niveaux : le paysage (macro) qui oriente les évolutions de la société sur le long terme, le régime sociotechnique (méso) où les routines organisent les activités ordinaires, et la niche (micro) où se développent les innovations radicales susceptibles de se diffuser au régime et de le changer. Le régime sociotechnique remplit une fonction (ex. le transport) grâce à la configuration d'éléments alignés (réseaux d'acteurs, règles et routines, artefacts). Il évolue de façon incrémentale pour résoudre ses problèmes, en sélectionnant les innovations. La niche favorise le développement de cette dernière en la protégeant temporairement, en l'encourageant et en l'émancipant [SMI 12]. Trois processus internes permettent à la niche de fonctionner : gestion des apprentissages, gestion des attentes et des visions, construction de réseaux [GEE 06b]. La dynamique d'une transition est présentée en figure 1.

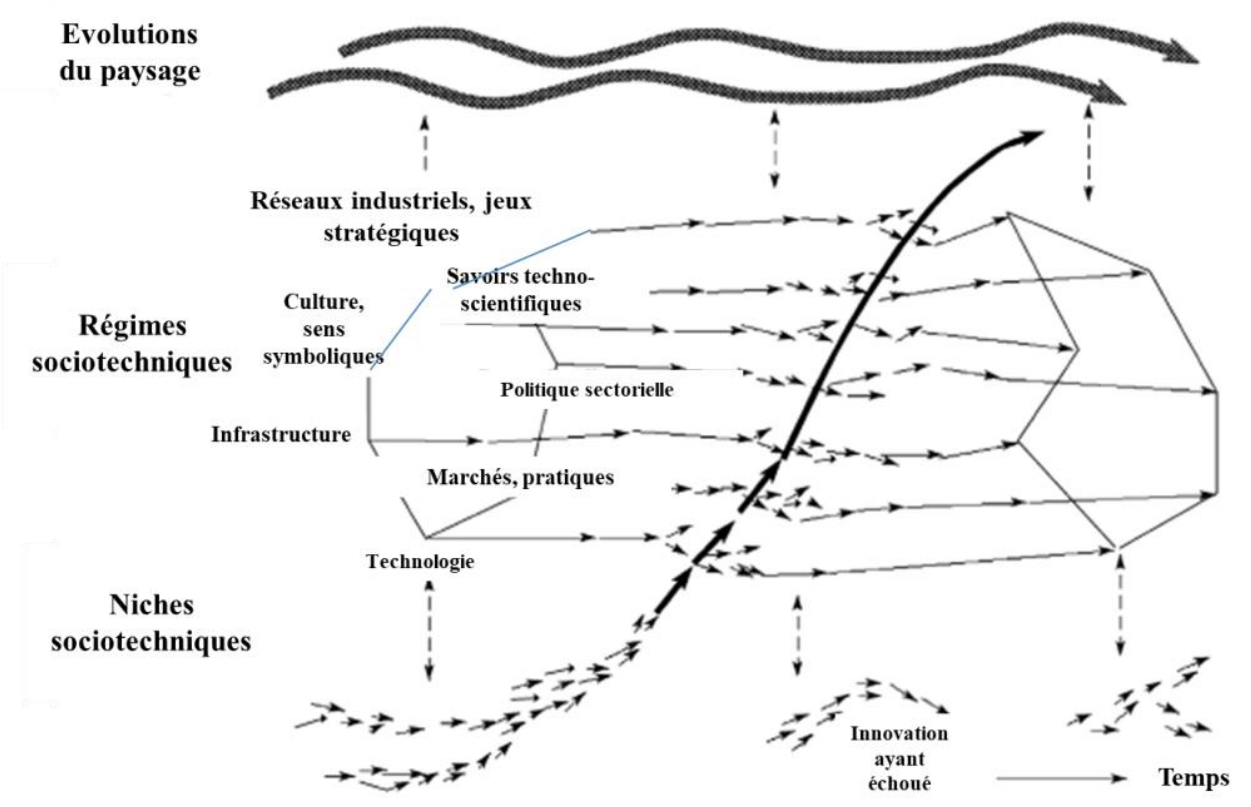

Figure 1. Dynamique de transition (d'après Geels, 2002) 
Une transition est un changement de régime sociotechnique [GEE 11] qui peut évoluer à partir d'une dynamique endogène, ou bien sous la pression du paysage ou de la niche. La niche peut se connecter au régime pour [SMI 12] de deux manières. Selon le processus fit and conform, elle devient compétitive et s'intègre au régime en alignant l'innovation avec les éléments du régime. Ou, selon le processus stretch and transform, les acteurs parviennent à changer des éléments d'un régime. Cela peut être en changeant les critères de sélection d'une innovation, en les complexifiant [HYY 15] ou en changeant les institutions. La niche se développe en lien avec le régime.

La transition doit aussi s'appréhender à partir de ses acteurs, de leurs stratégies et de leurs pratiques. Parmi les acteurs impliqués dans le changement, nous nous intéressons aux intermédiaires de transition.

\subsection{Les intermédiaires de transition}

Les intermédiaires de transition [KIV 14] [KIV 17] font l'objet d'un intérêt récent [BUS 17], et se présentent sous différentes formes : consultants [PON 10], communautés [HAR 13], organisations intermédiaires, agences gouvernementales [KIV 14]. Ils étendent le rôle des intermédiaires d'innovation [HOW 06] [AGO 17] dans une perspective systémique [VAN 03] [SMI 04]. Leurs interventions portent sur différents domaines simultanément (par exemple sur la demande et l'offre, sur la fiscalité et la réglementation). Ils focalisent leur action sur l'évolution des structures, des pratiques et des comportements.Ils participent à la création et au développement de la niche, tout en déstabilisant les éléments du régime [KIV 14]. Ils peuvent le faire de trois façons: en apportant des ressources, en activant les processus de la niche, en diffusant l'innovation. Leur intervention peut être permanente, épisodique ou contingente.

Premièrement, l'accent est mis sur leur rôle de courtier [KLE 09]. Ils apportent, à moindre coût, des ressources manquant aux acteurs [JOH 08] : financements [POL 16], connaissances [GEE 06a] [GEE 06b], réseaux [BOO 11] [AGO 17], légitimité [JOH 08]. Les intermédiaires agissant au niveau systémique peuvent faire davantage levier sur les ressources pour répondre à des enjeux environnementaux. Une innovation a un pouvoir de transformation d'autant plus grand qu'elle vise des changements au niveau du régime et que l'intermédiaire possède ses propres ressources [MOO 12]. Si un acteur local dispose de peu de ressources, il peut être limité dans sa capacité d'intervention et doit alors compter sur des acteurs privés [BUS 17] [TUR 12].

Deuxièmement, les intermédiaires de transition contribuent à la diffusion d'une innovation [HAR 13] en activant les processus de la niche. Pour diffuser une vision de la transition, ils construisent des réseaux et articulent la demande [STE 08]. Ils jouent un rôle de traducteurs [BOO 11] et d'interface (gatekeeper) entre les acteurs [POL 16]. La capacité d'un intermédiaire à diffuser largement une innovation tient en partie à sa capacité à faire circuler les savoirs [GEE 06a] [GEE 06b] et à s'adresser à des acteurs collectifs [MOO 12]. Ils sont à même d'intervenir au sein d'un système de relations complexes [VAN 03] [AGO 17].

Troisièmement, les intermédiaires de transition contribuent à la construction de réseaux et à la diffusion d'innovations par leur capacité à agir et à établir des liens entre différents 
niveaux et avec le régime [SMI 12]. Ils peuvent intervenir au sein d'un secteur ou d'un territoire, pour amener ce dernier vers une configuration systémique nouvelle et soutenable [KIV 17]. Ils peuvent aussi faire le lien [MOS 09] entre différents secteurs, et agir à différents niveaux de gouvernance (niveau local, régional ou national) [KIV 14, 17]. Un intermédiaire systémique peut faciliter le développement d'une innovation systémique, en agissant au niveau d'une chaîne de production, d'un secteur ou d'un ensemble de politiques publiques [KLE 09].

Enfin, les intermédiaires de transition interviennent selon différentes modalités. Un intermédiaire de transition peut agir de façon permanente (une agence gouvernementale dont ce serait la mission principale) [KIV 14] et relever d'une approche systémique [HOD 13] (par exemple favorisée par une nouvelle réglementation). L'intermédiation peut être épisodique autour d'un projet, ou d'une mission particulière [MOS 09]. Elle peut être contingente et émerger à partir des imperfections du système [JOH 08] : myopie des processus d'exploration vis-à-vis d'opportunités plus lointaines, des transferts de connaissances difficiles du fait de réseaux trop faibles, ou au contraire trop puissants, verrouillant tout changement.

Cinq types d'intermédiaires de transition peuvent être distingués [KIV 17] : 1) les intermédiaires systémiques interviennent à tous les niveaux, et adoptent une approche systémique du changement, 2) les intermédiaires du régime liés au régime existant (à travers des arrangements ou des intérêts institutionnels) sont mandatés pour promouvoir la transition, ce qui les amène à interagir avec des niches, 3) les intermédiaires de niche mènent ou promeuvent des expérimentations au sein d'une niche particulière, et cherchent à influencer le régime, 4) les intermédiaires de processus ou de projet facilitent un processus de changement ou un projet, promouvant ainsi les priorités d'acteurs extérieurs, 5) enfin, les intermédiaires d'utilisateurs traduisent les nouvelles technologies à l'intention des utilisateurs, les préférences des utilisateurs à l'intention des développeurs, et évaluent les technologies disponibles. Ils peuvent agir de façon complémentaire parfois en se substituant les uns aux autres en cas de défaillance [PON 10] [MAR 18], mais les intermédiaires systémiques et les intermédiaires de niche semblent essentiels pour une transition [KIV 17]. Les caractéristiques des intermédiaires de transition sont repris dans le tableau 1 : 


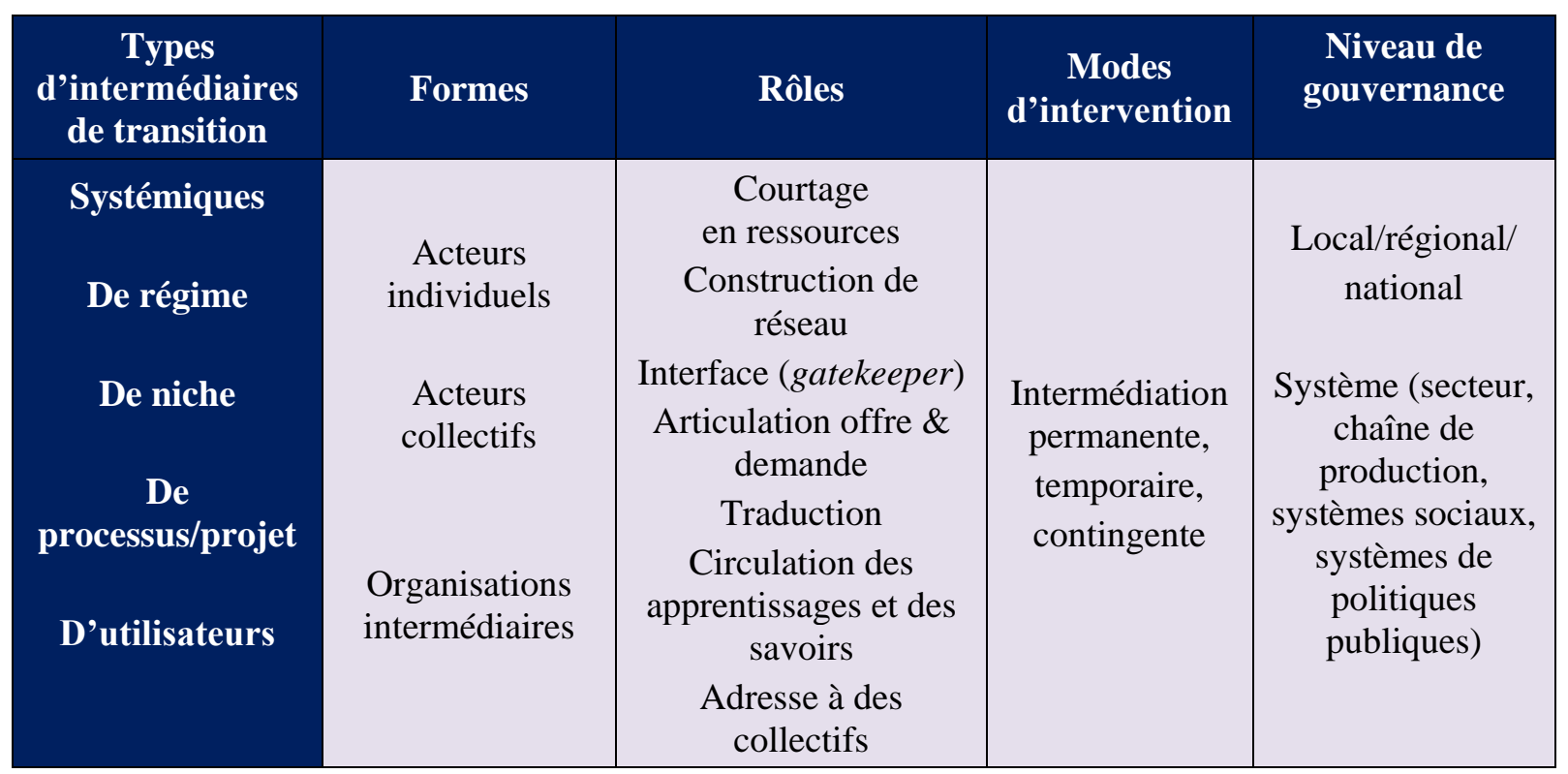

Tableau 1. Caractérisation des intermédiaires de transition

Les travaux sur les intermédiaires de transition montrent la diversité des figures d'intermédiation, la variété de leurs modes d'intervention. Le changement par l'innovation se faisant depuis la niche vers le régime [KEM 98] [GEE 02], il semble intéressant de prolonger les travaux récents en cherchant à caractériser les intermédiaires de transition engagés dans un projet d'innovation, et à comprendre les modalités d'émergence et d'intervention favorisant son développement.

\section{Méthodologie}

A cette fin, nous menons une recherche qualitative [DUM 13] autour de deux cas [RAG 92] d'innovation issus du secteur du recyclage en France. Les travaux dans ce secteur ont adopté une approche macro ou méso, mais le projet est rarement mobilisé comme unité d'analyse [BOL 18]. Or c'est bien à partir des innovations individuelles que se met en place une dynamique de transition. Après une phase exploratoire des enjeux du recyclage autour de l'innovation, plusieurs formes d'innovation sont apparues et nous avons retenu deux formes emblématiques : une innovation technologique soutenue par les politiques publiques et une innovation issue du secteur de l'économie sociale et solidaire. L'étude des deux projets d'innovation permet de mettre au jour des variables, lesquelles sont ensuite comparées dans les deux cas [BEC 16].

Le projet ValoMousse est une innovation créée sous l'impulsion de l'Ademe1 dans le cadre d'une nouvelle filière réglementaire (déchets d'ameublement). La collecte de données s'est principalement appuyée sur 14 entretiens semi-directifs, majoritairement en face à face et enregistrés (tableau 2), des documents écrits (rapports d'activités, brevets). Elle a été complétée par des sources secondaires (articles et interviews, presse et internet). La période observée s'étend de 2012 à 2015.

\footnotetext{
${ }^{1}$ Agence de l'Environnement et de la Maîtrise de l'Energie
} 


\begin{tabular}{|c|c|c|c|}
\hline Personnes \& entités & $\begin{array}{c}\text { Nombre } \\
\text { d'entretiens }\end{array}$ & Modalités & Durée totale \\
\hline $\begin{array}{c}\text { Ingénieur-innovateur } \\
\text { ValoMousse }\end{array}$ & 2 & Face à face (enregistrés) & $2 \mathrm{~h}$ \\
\hline $\begin{array}{l}\text { Business angel \& } \\
\text { administrateur }\end{array}$ & 1 & Face à face (enregistré) & $1 \mathrm{~h} 40$ \\
\hline Démanteleur & 1 & Face à face (non enregistré) & $1 \mathrm{~h}$ \\
\hline $\begin{array}{l}\text { Chargé de mission } \\
\text { Technopole }\end{array}$ & 2 & $\begin{array}{l}\text { Face à face (non } \\
\text { enregistrés) }\end{array}$ & $5 \mathrm{~h}$ \\
\hline Ingénieur Ademe & 1 & Téléphone (non enregistré) & $40 \mathrm{mn}$ \\
\hline $\begin{array}{c}\text { Industriel « sponsor » \& } \\
\text { client }\end{array}$ & 1 & Téléphone (non enregistré) & $45 \mathrm{mn}$ \\
\hline 2 Clients & 2 & $\begin{array}{c}1 \text { téléphone (non enregistré) } \\
1 \text { face à face (non } \\
\text { enregistré) }\end{array}$ & 45 mn chacun \\
\hline $\begin{array}{l}\text { Ingénieur fournisseur de } \\
\text { machine }\end{array}$ & 1 & Téléphone (enregistré) & $1 \mathrm{~h}$ \\
\hline $\begin{array}{l}\text { Chargé de mission } \\
\text { Comité de développement }\end{array}$ & 1 & Face à face (enregistré) & $1 \mathrm{~h}$ \\
\hline Chargée de mission CCI & 1 & Téléphone (non enregistré) & $30 \mathrm{mn}$ \\
\hline $\begin{array}{c}\text { Chargée de mission } \\
\text { Région }\end{array}$ & 1 & Téléphone (non enregistré) & $30 \mathrm{mn}$ \\
\hline
\end{tabular}

Tableau 2. Entretiens du projet ValoMousse

Le projet CircuPlastique est une innovation créée à l'initiative d'un entrepreneur social, hors filière réglementaire. De la même façon, les données ont été collectées par 14 entretiens (tableau 3), complétés de documents écrits ou vidéo comme sources secondaires (presse, internet). Nous avons participé à des réunions d'information organisées par l'innovateur où étaient invitées les parties prenantes du projet. La période observée s'étend de 2012 à 2016. 


\begin{tabular}{|c|c|c|c|}
\hline Personnes \& entités & $\begin{array}{c}\text { Nombre } \\
\text { d'entretiens }\end{array}$ & Modalités & Durée totale \\
\hline $\begin{array}{l}\text { Entrepreneur-innovateur } \\
\text { CircuPlastique }\end{array}$ & 1 & $\begin{array}{l}\text { Face à face } \\
\text { (enregistré) }\end{array}$ & $1 \mathrm{~h}$ \\
\hline $\begin{array}{l}\text { Chef de projet } \\
\text { CircuPlastique }\end{array}$ & 2 & $\begin{array}{c}\text { Face à face } \\
\text { (enregistré \& non } \\
\text { enregistré) }\end{array}$ & $1 \mathrm{~h} 30$ \\
\hline Enseignant Université & 2 & $\begin{array}{l}\text { Face à face (non } \\
\text { enregistrés) }\end{array}$ & $2 \mathrm{~h}$ \\
\hline $\begin{array}{l}\text { Chargés de mission } \\
\text { Technopole \& Agence } \\
\text { régionale d'innovation }\end{array}$ & 3 & $\begin{array}{c}\text { Face à face } \\
\text { (enregistrés et non } \\
\text { enregistré) }\end{array}$ & $3 h$ \\
\hline Ingénieur Ademe & 1 & $\begin{array}{l}\text { Téléphone (non } \\
\text { enregistré) }\end{array}$ & $30 \mathrm{mn}$ \\
\hline $\begin{array}{c}\text { Fondateurs cabinet } \\
\text { conseil innovation sociale }\end{array}$ & 2 & $\begin{array}{c}\text { Téléphone } \\
\text { (enregistré et non } \\
\text { enregistré) }\end{array}$ & $1 \mathrm{~h} 30$ \\
\hline $\begin{array}{l}\text { Ingénieur centre de } \\
\text { ressources technologiques }\end{array}$ & 1 & $\begin{array}{l}1 \text { face à face (non } \\
\text { enregistré) }\end{array}$ & $1 \mathrm{~h}$ \\
\hline $\begin{array}{l}\text { Chargé de mission } \\
\text { Métropole }\end{array}$ & 1 & $\begin{array}{l}\text { Téléphone (non } \\
\text { enregistré) }\end{array}$ & $30 \mathrm{mn}$ \\
\hline
\end{tabular}

Tableau 3. Entretiens du projet CircuPlastique

En nous appuyant en particulier sur la typologie de [KIV 17], nous cherchons ici à identifier et à caractériser les intermédiaires de transition impliqués autour d'un projet d'innovation, à comprendre leur mode d'émergence et d'intervention. Nous le faisons en analysant leurs apports en termes de ressources, leur rôle dans l'activation des processus de la niche (gestion des apprentissages, gestions des attentes et construction de réseaux).

Les cas, restitués sous forme de courtes monographies, sont construits à partir des données collectées, analysés individuellement et enfin discutés de façon croisée.

\section{Les enseignements issus de la création d'une filière de recyclage}

L'Ademe est l'opérateur public qui suit les objectifs nationaux de recyclage. Une feuille de route donne une vision à long terme du développement du recyclage. Sont identifiés des scénarios et les verrous technologiques, organisationnels, culturels [ADE 11]. Le secteur est déjà organisé en une vingtaine de filières, dotée d'éco-organismes, et impliquant producteurs, distributeurs, détenteurs de déchets et collectivités territoriales [ADE 17]. Un contrat d'objectifs, établi avec les collectivités locales et les collecteurs, est stimulé par une rémunération à la tonne recyclée par les éco-organismes. L'éco-organisme finance des opérations de R\&D pour améliorer les performances des filières de valorisation [ADE 17]. 


\subsection{Une nouvelle filière réglementaire dans le recyclage}

\subsubsection{L'émergence de la nouvelle filière réglementaire}

En 2010, l'Ademe étudie la création d'une nouvelle filière réglementaire (écoorganismes, éco-contribution) pour recycler les déchets de l'ameublement, qui sont en grande partie enfouis ou incinérés. De premières études sont lancées (préfiguration de la filière, état de l'art sur les techniques de recyclage du polyuréthane). L'Ademe agrée deux éco-organismes. Dans le même temps, l'agence lance un appel à manifestation d'intérêt (AMI) en 2011 pour faire émerger des expérimentations proches de la commercialisation. Il s'agit de mettre en place de nouveaux acteurs et des capacités de traitement pour atteindre les objectifs de recyclage. L'obligation faite aux producteurs de recycler doit créer un marché pour ces innovations.

Un ingénieur travaillant dans ce secteur est alerté par la dirigeante d'un cabinet conseil. Il collabore déjà à un plan de développement durable dans son usine. L'AMI permet de lancer son projet : fabriquer de nouveaux produits à partir de déchets et de chutes de production. Il veut créer ValoMousse, une unité de production complète dont il a déjà défini la stratégie. L'AMI impose un regroupement d'acteurs pour constituer une filière de valorisation : la présence de partenaires doit assurer l'accès au gisement en amont, et la commercialisation en aval. Il s'associe avec un démanteleur pour l'amont de la filière. Le cabinet conseil apporte des connaissances sur la mise sur le marché et les données environnementales. L'ingénieur pourra donc se concentrer sur la transformation de la matière. L'Ademe retient le projet car la filière proposée est complète, et permet de créer des capacités de recyclage. Elle introduit de la concurrence sur un marché où n'est présent qu'un seul transformateur issu d'une reconversion de site.

L'ingénieur doit créer son entreprise. Un chargé de mission de la technopole l'accompagne pour développer l'activité naissante, et s'investit énormément. Il aide à élaborer le modèle économique, des prestations d'études pouvant générer des revenus rapidement.

Dans le même temps, l'ingénieur bénéficie du soutien du fournisseur de machines, car l'innovation permet à ce dernier de surveiller la demande en matières recyclées. Le fournisseur le conseille dans la réalisation des prestations, met à disposition son laboratoire d'essais, l'équipe de techniciens pour la formulation de matières. Cette aide aboutit à des premiers prototypes que l'ingénieur peut montrer à ses prospects. Le fournisseur partage aussi son réseau de clients : certains deviennent clients et achètent des prestations, d'autres assurent la sous-traitance en attendant l'équipement de l'usine.

Du côté des utilisateurs, la demande émerge de deux façons. La nouvelle obligation réglementaire amène plusieurs grands industriels à trouver une solution de recyclage. Certains d'entre eux sont orientés par l'Ademe. D'autres clients, motivés par l'économie circulaire, cherchent à la mettre en œuvre, et le font parfois dans le cadre de projets de recherche. Ils s'engagent dans une forme de collaboration étroite, où ils apportent leurs actifs et la connaissances de leurs marchés respectifs (attentes des utilisateurs, réglementation) pour mettre au point les produits recyclés. Les brevets créés en copropriété sont commercialisés par les clients eux-mêmes sur leurs propres marchés, y compris à 
l'international. L'ingénieur peut ainsi tester et diffuser rapidement ses nouveaux produits. Le développement de l'innovation se heurte toutefois à des obstacles. La réglementation, par exemple, rend la traçabilité obligatoire mais celle-ci est impossible avec les déchets. Par ailleurs, certaines collaborations s'arrêtent parce que des clients refusent d'adapter leur production.

Pendant toute cette phase de prototypage, l'ingénieur rencontre des difficultés sur le plan financier. En attendant la réponse de l'Ademe à l'AMI, l'ingénieur a démarché les acteurs institutionnels, appuyé par un industriel local qui apporte une caution technique et morale. Très favorables à un projet industriel créateur d'emplois, les acteurs institutionnels conditionnent néanmoins leurs aides à l'accord de l'Ademe. De son côté, l'Ademe conditionne l'octroi de ses aides financières à l'accord des banques. Or celles-ci refusent de financer un projet à hauteur de plusieurs millions d'euros, qu'elles jugent trop important pour un porteur individuel. La situation est bloquée pendant plus d'un an. L'ingénieur doit trouver d'autres voies de financement car, contrairement au partenaire démanteleur qui collecte et traite déjà les déchets de la filière, il ne peut compter sur les aides financières des éco-organismes. Dans ce contexte, le fournisseur de machines assouplit ses conditions de paiement. L'accompagnateur suggère l'entrée au capital à des clients aux activités complémentaires et non concurrentes. Les démarches n'aboutissent pas mais donnent naissance à des co-brevets. Cette forme de co-propriété avec des acteurs installés protègera les actifs de l'entreprise si besoin. L'ingénieur démarche le réseau des anciens élèves où opère une structure d'investissement de type business angel. L'investisseur intéressé l'aide à démarcher des banques et, face à leur refus, provoque une médiation. Finalement, c'est la directrice du cabinet conseil, rentrée au capital de l'entreprise, qui fait intervenir une banque coopérative parisienne. Cet engagement débloque le financement des autres banques. Plusieurs levées de fonds ont lieu ensuite avec l'aide d'investisseurs institutionnels pour acquérir des machines et réaliser une opération de croissance externe.

\subsubsection{Une chaîne d'intermédiaires de transition incomplète autour de l'innovation}

En reprenant la typologie des intermédiaires de transition [KIV 17], on constate que la chaîne des intermédiaires est incomplète. Seulement deux catégories d'intermédiaires sont présentes. L'intermédiaire systémique, l'Ademe, est accompagné des cinq intermédiaires de projet (accompagnateur, industriel parrain, cabinet conseil, business angel et fournisseur de machines). Il manque l'éco-organisme, qui n'a aucune relation avec le projet qui concerne pourtant l'utilisation des matières recyclées. Alors qu'il est mandaté pour organiser la filière réglementaire, il n'intervient pas dans la création ni dans le développement de la niche. C'est l'Ademe, en tant qu'intermédiaire systémique qui remplit ce rôle: les décideurs publics lui ont donné mandat pour créer une niche (l'AMI) dans ce régime particulier.

Mais son action globale dépasse la seule aide à l'innovation et à la création d'une niche. Conformément à sa feuille de route, l'agence intervient à la fois en $R \& D$, dans la structuration des filières réglementaires et dans la production des connaissances associées. Aucun autre acteur ne recouvre complètement son champ d'action.

Sur six intermédiaires recensés, seuls deux sont engagés dans la transition. L'Ademe l'est par mission et le cabinet conseil en a fait son activité principale. Ils sont impliqués différemment. On peut émettre l'hypothèse, à ce stade, qu'un éco-organisme puisse être plus 
légitime et engagé dans le projet du fait de sa mission. Le cabinet conseil est plus impliqué dans le développement de l'activité sur les marchés et dans le capital. D'une certaine façon, ces deux intermédiaires de transition agissent sur les mêmes dimensions du projet (financement, marché), l'un au niveau macro (la filière), l'autre au niveau du projet (l'innovation). Les autres intermédiaires présents sont plus actifs dans le développement de la tehnologie et des produits, ce qui donne au projet une forte dimension industrielle.

On relève l'absence d'intermédiaires d'utilisateurs [KIV 17], ce qui peut limiter la diffusion ou l'adoption de l'innovation. Le travail de négociation et de clarification de la demande est assuré directement par l'ingénieur qui se retrouve face à des clients plus structurés que lui. Dans le cas présent, il se confronte à leurs exigences mais ne peut lever seul les obstacles identifiés, typiquement la réglementation ou les habitudes de production [GEE 02]. Ils ne peuvent pas être non plus levés par les autres intermédiaires du projet. Ainsi l'analyse de la chaîne d'intermédiaires présents fait apparaitre une relative fragilité du projet face aux difficultés rencontrées par l'innovation au moment de rentrer sur le marché. L'absence de soutien des banques aurait pu empêcher tout développement, sans l'intermédiation des partenaires du projet (investisseur et dirigeante du cabinet conseil).

On voit ici que l'accès à des ressources clés comme les financements [POL 16] est rendu possible parce que des intermédiaires de projet ont pallié un vide dans la chaîne d'intermédiation, et se sont substitués localement à des intermédiaires de processus en retrait (banques) [PON 10]. L'Ademe accorde une aide financière essentielle au départ, puis intervient ponctuellement, en conservant une grande neutralité vis-à-vis des acteurs du marché [KIV 14]. Son mandat lui permet de créer une niche en vue de la transition, mais pas d'agir quand apparaissent les difficultés. Ce vide fait émerger les intermédiaires de projet : ils se mobilisent car ils veulent que l'innovation réussisse. Chacune de leurs interventions ou apports devient alors critique [JOH 08] : installations techniques, connaissances, réseau. Chaque intermédiaire a agi de sa propre initiative. Ceci montre en creux la présence nécessaire et l'implication de ce type d'intermédiaires pour que l'innovation ait une chance de réussir.

L'innovation se développe ici dans un schéma fit and conform. L'Ademe aligne les éléments nécessaires à la transition à travers la niche et les actions au niveau du régime (réglementation, éco-organismes) et suit la vision décrite dans sa feuille de route. Individuellement chaque intermédiaire puise dans ses routines, son réseau et ses connaissances. A travers leur rôle de courtier [KLE 09], les intermédiaires mettent en place des éléments favorables et alimentent les processus de la niche.

Face aux besoins ou difficultés du projet, ils encouragement l'innovation en lui apportant les ressources et en construisant les réseaux nécessaires à ce stade (clients co-propriétaires de brevets, banques). Ils permettent de générer des apprentissages autour de l'innovation. L'ingénieur les absorbe rapidement. Certains clients contribuent directement à la diffusion de l'innovation en commercialisant les produits sur leurs marchés.

Le tableau 4 présente les intermédiaires présents autour de ValoMousse, et les modalités de leurs interventions. 


\subsection{L'initiative d'un entrepreneur social pour créer de l'emploi}

\subsubsection{L'émergence d'une nouvelle activité autour d'un gisement négligé}

Le projet d'innovation est porté par un entrepreneur social qui a créé une entreprise adaptée. Il veut favoriser un changement sociétal et mener une innovation sociale matérialisée à travers son entreprise. De son côté, la Métropole, engagée dans l'économie circulaire, veut recycler ses déchets de bureaux. Le recyclage de papiers réunit les deux projets. Après quelques années d'activité, l'entrepreneur anticipe la baisse des aides publiques et veut diversifier ses revenus. Il s'intéresse à une solution de recyclage pour la vaisselle plastique jetable (tri et de nettoyage) développée à l'université. Ce gisement est négligé par les grandes entreprises car il est diffus et peu rentable et qu'il n'y a pas de filière réglementaire. La dimension technologique n'intéresse pas l'entrepreneur, qui veut créer des emplois et changer les comportements. Cette invention peut aussi permettre de matérialiser l'intérêt des acteurs pour le recyclage, qu'il informe rapidement. L'entrepreneur bénéficie du soutien d'un accompagnateur institutionnel qui l'aide à optimiser les aides financières, mais peu au-delà car l'entrepreneur possède de solides compétences en gestion et a un réseau étendu. Il sollicite des financements auprès des institutionnels. Pour l'Ademe, cette nouvelle technologie permet de créer des connaissances nouvelles sur le recyclage des plastiques, potentiellement applicables à d'autres plastiques. Elle participe au financement de la machine au titre de la «plus-value environnementale », malgré la future interdiction de la vaisselle jetable et les faibles tonnages concernés. L'éco-organisme de la filière pense trouver une solution pour d'autres emballages dans le cadre de sa propre stratégie. Deux acteurs de la finance solidaire acceptent de soutenir le développement de l'entreprise, avec le projet. Ce sont les contributeurs principaux. Les institutions régionales ont plus de difficultés à adapter leurs aides au projet d'innovation qui répond à des enjeux sociaux et environnementaux.

Pour la partie technologique, l'entrepreneur engage un bureau d'études et un ergonome. Il veut préserver la cohérence entre projet d'entreprise, développement d'activité et gains environnementaux. La matière obtenue doit être hautement recyclable, une partie des opérations doit rester manuelle pour créer des postes de travail et augmenter les compétences des employés, réduire la consommation d'eau et d'énergie. Ces pré-requis se combinent pourtant mal avec les habitudes de conception du bureau d'études, qui ne les intègre pas. L'entrepreneur change de prestataire, mais la dimension technologique prend de plus en plus d'importance. Finalement, l'entrepreneur arbitre en faveur du tri manuel, plus performant que les machines et qui crée des postes. L'ergonome traduit cette ambition dans la conception de la machine. Le tri manuel permet de trier des matières différentes, et l'une peut être financée par l'éco-organisme de la filière. Finalement, les quantités triées, plus importantes que prévues, permettent d'envisager une progression de l'activité satisfaisante à l'échelle de l'entreprise.

L'entrepreneur confie l'analyse de la demande à un centre de ressources technologiques régional positionné sur l'économie circulaire. Les plasturgistes sont intéressés, mais veulent des échantillons pour se prononcer sur la qualité. Le centre de ressources fournit à l'entrepreneur une liste d'utilisateurs potentiels. Il explore ensuite des applications possibles. Il faut rapidement sécuriser l'accès au gisement pour collecter les quantités nécessaires. La Métropole contribue activement à la constitution d'une filière de collecte, en 
tirant profit de sa capacité d'action rapide, à grande échelle. Ce projet illustre pour elle l'économie circulaire. Elle mobilise des acteurs publics et privés pour créer des synergies locales, et veut contribuer à l'effet d'entraînement du projet et à la sensibilisation à sa thématique. Les quantités de déchets collectées sont suffisantes pour assurer la fourniture de la future machine. De son côté, l'entrepreneur entame la recherche de contrats de collecte avec les grands sites tertiaires du territoire. En parallèle, l'entrepreneur entame un travail pour changer leurs comportements des distributeurs.

La clé de voute du projet est l'implication d'un réseau d'acteurs autour du projet. Pour en assurer la résilience, en cas de départ de l'un d'eux, l'entrepreneur souhaite identifier les parties prenantes de son projet, au titre de la faisabilité. Cet argument est défendu par l'accompagnateur institutionnel auprès de la banque publique d'investissement, qui accepte de changer ses critères et de financer l' « étude de renforcement du projet » (entrepreneur). Tout au long du développement du projet, l'entrepreneur organise des «comités de pilotage » et des réunions d'information collective à destination des parties prenantes. C'est un vecteur pour les impliquer dans un enjeu social plus large que la machine elle-même. Un collectif émerge. Pour appuyer sa démarche globale, l'entrepreneur missionne un cabinet conseil, spécialisé en innovation sociale, pour définir un système de mesure de l'impact social de son action, qui aura vocation à être utilisé pour d'autres projets.

\subsubsection{Des intermédiaires de transition bien représentés autour de l'innovation}

La chaîne d'intermédiaires résulte d'un travail de composition de l'entrepreneur. Les intermédiaires émergent ici en fonction de ses choix stratégiques. Dans le projet CircuPlastique, les intermédiaires constituent une chaîne assez complète autour de l'innovation. Toutes les catégories d'intermédiaires sont représentées (tableau 5) [KIV 17] : l'Ademe en tant qu'intermédiaire systémique, des intermédiaires de niche (Métropole, centre de ressources technologiques) et de projet (cabinet conseil en innovation sociale, accompagnateur institutionnel). Ici, l'intermédiaire de régime, l'éco-organisme de la filière concernée, est également impliqué.

Les intermédiaires se caractérisent par leur lien avec la transition, sans pourtant agir avec le même engagement. L'Ademe, comme l'éco-organisme, interviennent ici à la demande de l'entrepreneur, ce que nous appelons émergence passive, motivés par les connaissances qui pourront être mobilisées dans d'autres filières ou d'autres produits. L'éco-organisme intervient plus tard dans le projet, en finançant les tonnes qui seront effectivement recyclées. Les intermédiaires systémiques et de régime alimentent peu les processus de la niche : leur contribution financière intervient $a$ posteriori ou de façon moins importante que les acteurs de la finance solidaire. Les intermédiaires réellement engagés dans la transition (par mandat ou par choix) oeuvrent au changement des pratiques environnementales ou sociales, à la diffusion des connaissances vers de futurs projets, dans leurs domaines d'intervention respectifs. Ici, ces intermédiaires correspondent bien aux intermédiaires décrits par [GEE 06a].

Les interventions des différents types d'intermédiaires sont aussi complémentaires et permettent de développer l'innovation dans ses différentes dimensions (sociales, environnementales, techniques). En aval, le rôle d'intermédiaire d'utilisateurs est assuré par le centre de ressources technologiques, qui traduit les attentes des plasturgistes auprès de 
l'entrepreneur. Il entame le travail d'articulation entre l'offre de matière recyclée et la demande [VAN 03]. Une deuxième catégorie d'utilisateurs, les futurs opérateurs de la machine, est représentée par l'ergonome qui traduit leurs besoins [SMI 07] [BOO 11] aux concepteurs. En amont, la Métropole assure à l'entrepreneur une ressource critique (gisement) et contribue à la légitimité et à la diffusion de l'innovation par des mises en réseau et des synergies.

La niche initiée par l'entrepreneur se développe dans une dynamique de stretch and transform. [SMI 12]. C'est donc lui qui ordonne la temporalité des interventions et change d'intermédiaires si ceux-ci ne s'alignent pas sur ses objectifs. Les intermédiaires impliqués sur la partie technologique (l'ergonome et les bureaux d'études) travaillent de façon plus ponctuelle et circonscrite, la technique étant moins importante aux yeux de l'entrepreneur. L'entrepreneur cherche à changer les critères de sélection du régime (financement d'une étude de faisabilité sur des critères inédits, promotion d'une mesure de l'impact social avec le cabinet conseil).

En s'affranchissant des critères traditionnels de sélection du régime, l'entrepreneur sélectionne à son tour les intermédiaires qui contribueront au développement de son innovation. Dans cette dynamique, les intermédiaires apportent des ressources qui soutiennent et catalysent les processus de la niche qui sont activés par l'entrepreneur. Ainsi la protection est rendue possible à travers l'étude de faisabilité qui permet de mesurer la dépendance du projet vis-à-vis des parties prenantes. Le financement solidaire apporte à la fois une protection et de l'encouragement, tout comme la Métropole qui assure l'approvisionnement de la matière. C'est également l'entrepreneur qui crée les conditions de l'émancipation de l'innovation (changement des critères de sélection, choix des partenaires, création d'une arène et d'un réseau).

Le tableau 5 présente les intermédiaires présents autour de CircuPlastique, et les modalités de leurs interventions. 


\begin{tabular}{|c|c|c|c|c|c|c|c|}
\hline $\begin{array}{l}\text { Intermédiaire de } \\
\text { transition }\end{array}$ & Type & $\begin{array}{c}\text { Mission } \\
\text { principale } \\
\text { de transition }\end{array}$ & Engagement & $\begin{array}{c}\text { Mode } \\
\text { d'émergence }\end{array}$ & Ressources & $\begin{array}{l}\text { Processus de la } \\
\text { niche }\end{array}$ & $\begin{array}{l}\text { Dynamique } \\
\text { d'innovation }\end{array}$ \\
\hline Ademe & Systémique & Oui & $\begin{array}{l}\text { Episodique } \\
\text { (projet) et } \\
\text { permanent } \\
\text { (transition) }\end{array}$ & Mandat & $\begin{array}{c}\text { Financement } \\
\text { Orientation des } \\
\text { demandes des } \\
\text { clients }\end{array}$ & $\begin{array}{c}\text { Gestion des } \\
\text { attentes } \\
\text { Construction de } \\
\text { réseaux }\end{array}$ & \multirow{6}{*}{$\begin{array}{c}\text { Innovation } \\
\text { technologique } \\
\text { (nouveau } \\
\text { procédé, } \\
\text { nouveaux } \\
\text { produits et } \\
\text { usages) } \\
\\
\text { Innovation } \\
\text { organisationnelle } \\
\text { (nouvelle filière } \\
\text { de recyclage) }\end{array}$} \\
\hline Accompagnateur & De projet & Non & Permanent & Mandat & $\begin{array}{c}\text { Connaissances } \\
\text { Réseau }\end{array}$ & $\begin{array}{l}\text { Apprentissage } \\
\text { Construction de } \\
\text { réseaux }\end{array}$ & \\
\hline $\begin{array}{l}\text { Industriel } \\
\text { «parrain » }\end{array}$ & De projet & Non & Permanent & Contingent & $\begin{array}{c}\text { Légitimité } \\
\text { Réseau } \\
\text { Actifs }\end{array}$ & $\begin{array}{l}\text { Gestion des } \\
\text { attentes } \\
\text { Apprentissage } \\
\text { Construction de } \\
\text { réseaux }\end{array}$ & \\
\hline $\begin{array}{l}\text { Cabinet conseil } \\
\text { (partenaire et } \\
\text { actionnaire) }\end{array}$ & De projet & Oui & Permanent & Contingent & $\begin{array}{c}\text { Connaissances } \\
\text { Financement } \\
\text { Réseau }\end{array}$ & $\begin{array}{c}\text { Gestion des } \\
\text { attentes } \\
\text { Apprentissage } \\
\text { Construction de } \\
\text { réseaux }\end{array}$ & \\
\hline Business Angels & De projet & Non & Permanent & Contingent & $\begin{array}{c}\text { Connaissances } \\
\text { Financement } \\
\text { Réseau }\end{array}$ & $\begin{array}{c}\text { Gestion des } \\
\text { attentes } \\
\text { Apprentissage } \\
\text { Construction de } \\
\text { réseaux }\end{array}$ & \\
\hline $\begin{array}{l}\text { Fournisseur de } \\
\text { machines }\end{array}$ & De projet & Non & Permanent & Contingent & $\begin{array}{c}\text { Connaissances } \\
\text { Actifs } \\
\text { Réseau }\end{array}$ & $\begin{array}{l}\text { Apprentissage } \\
\text { Construction de } \\
\text { réseaux }\end{array}$ & \\
\hline
\end{tabular}

Tableau 4. Caractérisation et modalités d'intervention des intermédiaires autour de ValoMousse 


\begin{tabular}{|c|c|c|c|c|c|c|c|}
\hline $\begin{array}{l}\text { Intermédiaire } \\
\text { de transition }\end{array}$ & $\begin{array}{c}\text { Type } \\
\text { d'intermédiaire }\end{array}$ & $\begin{array}{c}\text { Mission } \\
\text { principale de } \\
\text { transition }\end{array}$ & Engagement & $\begin{array}{c}\text { Mode } \\
\text { d'émergence }\end{array}$ & Ressources & $\begin{array}{c}\text { Processus de la } \\
\text { niche }\end{array}$ & $\begin{array}{l}\text { Dynamique } \\
\text { d'innovation }\end{array}$ \\
\hline Ademe & Systémique & Oui & $\begin{array}{l}\text { Episodique } \\
\text { (projet) } \\
\text { et permanent } \\
\text { (transition) }\end{array}$ & $\begin{array}{c}\text { Emergence } \\
\text { passive (réponse } \\
\text { à une demande) }\end{array}$ & Financement & & \multirow{7}{*}{$\begin{array}{c}\text { Innovation } \\
\text { technologique } \\
\text { (nouvelle machine) } \\
\\
\text { Innovation } \\
\text { organisationnelle } \\
\text { (nouvelle filière) } \\
\text { et technologique } \\
\text { (machine adaptée } \\
\text { aux salariés et aux } \\
\text { enjeux } \\
\text { environnementaux) } \\
\text { Innovation } \\
\text { institutionnelle } \\
\text { (nouveaux critères } \\
\text { de sélection des } \\
\text { innovations) }\end{array}$} \\
\hline Eco-organisme & De régime & Oui & Episodique & $\begin{array}{c}\text { Emergence } \\
\text { passive } \\
\text { (financement en } \\
\text { fin du } \\
\text { processus) }\end{array}$ & Financement & & \\
\hline Métropole & De niche & $\begin{array}{c}\text { Non (mandat) } \\
\text { Oui } \\
\text { (économie } \\
\text { circulaire) }\end{array}$ & Permanent & Contingent & $\begin{array}{l}\text { Légitimité } \\
\text { Actifs }\end{array}$ & $\begin{array}{c}\text { Gestion des } \\
\text { attentes } \\
\text { Construction de } \\
\text { réseau }\end{array}$ & \\
\hline $\begin{array}{l}\text { Centre régional } \\
\text { de ressources } \\
\text { technologiques }\end{array}$ & De niche & Oui & Episodique & Contractuelle & $\begin{array}{c}\text { Connaissances } \\
\text { Réseau }\end{array}$ & $\begin{array}{c}\text { Gestion des } \\
\text { attentes } \\
\text { Apprentissages } \\
\text { Construction de } \\
\text { réseau } \\
\end{array}$ & \\
\hline $\begin{array}{c}\text { Cabinet conseil } \\
\text { en innovation } \\
\text { sociale }\end{array}$ & De projet & Oui & Permanent & Contractuelle & Connaissances & $\begin{array}{l}\text { Gestion des } \\
\text { attentes }\end{array}$ & \\
\hline $\begin{array}{l}\text { Accompagnateur } \\
\text { institutionnel }\end{array}$ & De projet & Non & Permanent & Mandatée & Connaissances & $\begin{array}{c}\text { Gestion des } \\
\text { attentes } \\
\text { Apprentissages }\end{array}$ & \\
\hline Ergonome & D'utilisateurs & Non & Episodique & Contractuelle & Connaissances & $\begin{array}{c}\text { Gestion des } \\
\text { attentes }\end{array}$ & \\
\hline
\end{tabular}

Tableau 5. Caractérisation et modalités d'intervention des intermédiaires autour de CircuPlastique 


\section{Discussion : le rôle des intermédiaires de transition dans les dynamiques d'innovation}

Nous avons cherché à approfondir les travaux sur les intermédiaires de transition [KIV 14], en nous interrogeant sur leur rôle dans le développement d'une niche sociotechnique. La comparaison de deux cas distincts [BEC 16] permet de faire apparaittre un lien possible entre la dynamique de la niche (fit and conform ou stretch and transform) [SMI 12] et les modalités de l'intermédiation systémique.

Premièrement, l'intermédiation systémique [HOD 13] peut s'envisager à travers une chaîne d'intermédiaires présents autour de l'innovation [PON 10], plutôt qu'à partir de la présence d'un intermédiaire systémique [VAN 03]. Dans ce cas, une chaîne peut se caractériser par sa complétude ou son incomplétude. Dans le projet ValoMousse, les intermédiaires systémiques et de niche sont présents, tandis que manquent à l'appel plusieurs autres types d'intermédiaires [KIV 17]. Les intermédiaires de régime (les éco-organismes) sont absents alors qu'il s'agit de changer le régime. De même, les intermédiaires d'utilisateurs ne permettent pas de traduire les attentes du marché alors qu'il s'agit de multiplier les usages. Dans le projet CircuPlastique, les intermédiaires de transition forment une chaîne complète: présence des intermédiaires systémiques, de régime, de niche. Les intermédiaires de processus (banques) apparaissent ici comme des acteurs critiques. Le projet ValoMousse est bloqué tant que l'accès au financement n'est pas résolu par d'autres intermédiaires de la chaîne. On peut donc envisager un lien entre la complétude de la chaîne d'intermédiaires et la réussite de l'innovation. Celle-ci pourrait être conditionnée à la capacité des intermédiaires à se substituer aux intermédiaires défaillants, grâce à leurs ressources et à leur motivation. Se pose en creux la question de la solidarité entre eux. L'intermédiation systémique peut aussi être assurée par une chaîne composée d'intermédiaires non systémiques.

Deuxièmement, notre recherche apporte quelques nuances aux travaux actuels [KIV 17, 18] [MAR 18]. Les cas éclairent l'importance de certains intermédiaires : il est alors utile d'examiner la composition de la chaîne. Dans le secteur du recyclage, les intermédiaires d'utilisateurs contribuent à la diffusion et à l'adoption de l'innovation, en particulier si l'enjeu est l'ouverture de la boucle de la matière. Ses enjeux spécifiques, bien différents des secteurs plus étudiés comme l'énergie ou le transport, permettent de montrer les spécificités de la niche, où il est nécessaire de prendre en compte l'amont et l'aval d'une innovation, comme le fait l'Ademe dans le cadre de l'AMI (la filière complète de valorisation).

Les difficultés de ValoMousse montrent combien l'accès aux financements est critique [POL 16], assuré finalement par des intermédiaires de niche quand l'intermédiaire systémique ne peut pas débloquer la situation. On voit que l'intermédiaire systémique peut être présent autour de la niche tout en soutenant l'innovation de façon limitée (l'Ademe dans le cas CircuPlastique). Ceci amende les conclusions de [KIV 17].

Troisièmement, la composition de la chaîne d'intermédiation peut découler des choix stratégiques faits par l'innovateur. Il développe son innovation en s'alignant avec les objectifs du régime, comme dans le cas de ValoMousse. Il est accompagné de façon traditionnelle pour la création d'entreprise. Au contraire CircuPlastique se développe dans une niche en marge du régime. Les intermédiaires sollicités partagent les mêmes valeurs que l'innovateur et la place relative des intermédiaires de niche est centrale. Cela peut être en partie relié au fait que la niche 
(stretch and transform) est créée à l'initiative de l'entrepreneur, et non d'un intermédiaire du régime ou systémique. cela tient surtout au management de l'innovation menée par l'entrepreneur. Un entrepreneur ayant une sensibilité différente (comme dans le cas ValoMousse) peut choisir de laisser jouer le rôle principal aux intermédiaires traditionnels dans une dynamique fit and conform [SMI 12].

Ce travail sur les intermédiaires de transition peut se prolonger en liant intermédiation de transition et business model. Il est intéressant de comprendre comment l'intermédiation systémique, à travers les intermédiaires de transition, peut aider à élaborer des business models durables, des business models circulaires nécessitant une approche systémique. Les éléments apportés permettraient d'orienter les politiques publiques ou les gestionnaires de l'innovation.

\section{Bibliographie}

[ADE 11] ADEME, Collecte, tri, recyclage et valorisation des déchets, Feuille de route stratégique. 2011.

[ADE 17] ADEME, Les filières à responsabilité élargie du producteur, Panorama. 2017.

[AGO 17] AgoguÉ M., Berthet E., FredBerg T., et al. «Explicating the role of innovation intermediaries in the "unknown": A contingency approach". Journal of Strategy and Management, vol. 10, n 1, p. 19-39, 2017.

[BEC 16] BECKER H., La bonne focale : de l'utilité des cas particuliers en sciences sociales, La Découverte, Paris, 2016.

[BOL 18] BOLDRINI J.-C., «La co-création de valeur dans un projet d'innovation collaboratif : un cas de transition vers l'économie circulaire », Innovations, $\mathrm{n}^{\circ}$ 1, p. 143-171, 2018.

[BOO 11] BoON W.PC, MoORS E.HM, KuHLMANN S., et al., "Demand articulation in emerging technologies: intermediary user organisations as co-producers?", Research Policy, vol. 40, n 2, p. 242-252, 2011.

[BUS 17] Bush R.E., BALE C. SE, Powell M., et al., " The role of intermediaries in low carbon transitionsEmpowering innovations to unlock district heating in the UK", Journal of cleaner production, vol. 148, p. 137$147,2017$.

[DUM 13] DUMEZ H., Méthodologie de la recherche qualitative, Vuibert, Paris, 2013.

[GEE 02] GEELS F. W., "Technological transitions as evolutionary reconfiguration processes: a multi-level perspective and a case-study", Research policy, vol. 31, n 8-9, p. 1257-1274, 2002.

[GEE 06a] GEELS F., DEUTEN J. J., "Local and global dynamics in technological development: a socio-cognitive perspective on knowledge flows and lessons from reinforced concrete", Science and Public Policy, vol. 33, $\mathrm{n}^{\circ} 4$, p. 265-275, 2006a.

[GEE 06b] GEELS F., RAVEN R., "Non-linearity and expectations in niche-development trajectories: ups and downs in Dutch biogas development (1973-2003)", Technology Analysis \& Strategic Management, vol. 18, $\mathrm{n}^{\circ}$ 3-4, p. 375-392, 2006b.

[GEE 11] GEELS F. W., "The multi-level perspective on sustainability transitions: Responses to seven criticisms", Environmental innovation and societal transitions, vol. 1, n 1, p. 24-40, 2011.

[HAR 13] Hargreaves T., Hielscher S., Seyfang G., et al., « Grassroots innovations in community energy: The role of intermediaries in niche development", Global environmental change, vol. 23, ${ }^{\circ}$ 5, p. 868-880, 2013. 
[HOD 13] Hodson M., MARVIN S., BUlKeley H., "The intermediary organisation of low carbon cities: a comparative analysis of transitions in Greater London and Greater Manchester", Urban Studies, vol. 50, $\mathrm{n}^{\circ} 7$, p. 1403-1422, 2013.

[HOW 06] HowELLS J., "Intermediation and the role of intermediaries in innovation", Research policy, vol. 35, $\mathrm{n}^{\circ} 5$, p. 715-728, 2006.

[HYY 15] HYYTINEN K., TOIVONEN M., "Future energy services: empowering local communities and citizens", Foresight, vol. 17, n 4, p. 349-364, 2015.

[JOH 08] JOHNSON W. HA., "Roles, resources and benefits of intermediate organizations supporting triple helix collaborative R\&D: The case of Precarn", Technovation,vol. 28, n 8, p. 495-505, 2008.

[KEM 98] KEMP R., SCHOT J., HOOGMA R., "Regime shifts to sustainability through processes of niche formation: the approach of strategic niche management", Technology analysis \& strategic management, vol. $10, \mathrm{n}^{\circ} 2$, p. $175-198,1998$.

[KIV 14] KIVIMAA P., "Government-affiliated intermediary organisations as actors in system-level transitions", Research policy, vol. 43, n 8, p. 1370-1380. 2014.

[KIV 17] KIVIMAA P., BOON W., HyYSAlO S., et al., Towards a typology of intermediaries in transitions: a systematic review. 2017, SPRU Working Paper Series (SWPS), 2017-17: 1-27. ISSN 2057-6668. www.sussex.ac.uk/spru/swps2017-17

[KIV 18] KIVIMAA P., MARTISKAINEN M., "Dynamics of policy change and intermediation: the arduous transition towards low-energy homes in the United Kingdom”, Energy Research \& Social Science, vol. 44, p. 83-99, 2018.

[KLE 09] KLERKX L., LEUUWIS C., "Establishment and embedding of innovation brokers at different innovation system levels: Insights from the Dutch agricultural sector", Technological forecasting and social change, vol. 76, n 6, p. 849-860, 2009.

[MAR 18] MARTISKAINEN M., KIVIMAA P., "Creating innovative zero carbon homes in the United KingdomIntermediaries and champions in building projects", Environmental Innovation and Societal Transitions, vol. 26, p.15-31, 2018.

[MOO 12] MOORE M.-L., WeSTley F. R., BrodHEAd T., "Social finance intermediaries and social innovation", Journal of Social Entrepreneurship, vol. 3, n 2, p. 184-205, 2012.

[MOS 09] MOSS T., "Intermediaries and the governance of sociotechnical networks in transition", Environment and Planning A, vol. 41, n 6 , p. 1480-1495, 2009.

[POL 16] POLZIN F., VON FLOTOW P., KLERKX, L., “Addressing barriers to eco-innovation: Exploring the finance mobilisation functions of institutional innovation intermediaries", Technological Forecasting and Social Change, vol. 103, p. 34-46, 2016.

[PON 10] PONCET J., KUPER M., CHICHE J., "Wandering off the paths of planned innovation: The role of formal and informal intermediaries in a large-scale irrigation scheme in Morocco", Agricultural systems, vol. 103, n 4, p. 171-179, 2010.

[RAG 92] RAGIN C., BECKER H. S. (ed.), "What is a case?: exploring the foundations of social inquiry”, Cambridge university press, 1992.

[SMI 04] SMITS R., KUHLMANN S., "The rise of systemic instruments in innovation policy”, International journal of foresight and innovation policy, vol. 1, $\mathrm{n}^{\circ} 1-2$, p. 4-32, 2004.

[SMI 12] SMITH A., RAVEN R., "What is protective space? Reconsidering niches in transitions to sustainability", Research policy, vol. 41, n 6, p. 1025-1036, 2012. 
[STE 08] STEWART J., Hyysalo S., "Intermediaries, users and social learning in technological innovation", International Journal of Innovation Management, vol. 12, nº 03, p. 295-325, 2008.

[TUR 12] TURNHEIM B., GEELS F. W., "Regime destabilisation as the flipside of energy transitions: Lessons from the history of the British coal industry (1913-1997)", Energy Policy, vol. 50, p. 35-49, 2012.

[VAN 03] VAn Lente H., HeKKeRt M., SMits R., et al., "Roles of systemic intermediaries in transition processes", International journal of Innovation management, vol. 7, n 03, p. 247-279, 2003. 